\title{
Two-Parameter Inversion of Fluid-Saturated Porous Medium with Niche Ant Colony Algorithm
}

\author{
Xin-Ming Zhang and Li-Rong Wang \\ Harbin Institute of Technology Shenzhen Graduate School, Shenzhen, Guangdong 518055, China \\ Correspondence should be addressed to Xin-Ming Zhang; xinmingxueshu@gmail.com
}

Received 6 November 2013; Accepted 2 December 2013; Published 9 January 2014

Academic Editor: Manuel Ruiz Galan

Copyright ( 2014 X.-M. Zhang and L.-R. Wang. This is an open access article distributed under the Creative Commons Attribution License, which permits unrestricted use, distribution, and reproduction in any medium, provided the original work is properly cited.

\begin{abstract}
Based on the Biot model, we performed the inversion of reservoir parameters with improved niche ant colony algorithms (INACA). In order to overcome the premature problem of inverse process, the improved niche ant colony algorithms are constructed by combining the fitness-sharing principle which is one of the niche methods with the ant colony algorithm. The numerical results indicate that the relative error of single parameter inversion can be maintained at less than 0.4 percent; particulary, the relative error of porosity is less than 0.02 percent. However, the inversion effect of two-parameter inversion was found to be slightly weak, but the relative error can still be maintained at less than 7.5 percent. Moreover, the antinoise property of the method was also verified. The results of numerical simulation demonstrate that the method is an effective convergent optimization method.
\end{abstract}

\section{Introduction}

The fluid-saturated porous medium is viewed herein as a two-phase system consisting of a solid and a fluid phase, each of which is regarded as a continuum, and each is following its own motion. Biot $[1,2]$ first formulated linear governing equations for the interaction of two such phases in the 1950s. Compared with the classical single-phase elastic medium theory, the fluid-saturated porous medium wave equation is more complicated and in the model there are many coefficients which represent rock and flow properties. Therefore, the fluid-saturated porous medium theory describes the actual earth stratum more precisely and has much more trouble in parameter estimation process.

According to the fact that the computed response should fit with the measured one, the parameter estimation can be reduced to solve an optimum problem. The traditional local optimum methods and iterative linear methods such as gradient method, perturbation method, and time convolution regularization iterative method are based on Newton iterative method; it is with local convergence. On the contrary, the global optimal methods, such as genetic algorithm, simulated annealing method, and ant colony algorithm, do not require more for the objective function in the general function optimization problems. Thus, global optimal methods have recently attracted the interest of numerous scholars. Meanwhile, as a newly developed global optimal method, the ant colony algorithm has also captured the interest of many scholars. The original ant colony algorithm was proposed by Colorni et al. [3] in 1991. This algorithm was an optimization methodology based on the foraging behavior of Argentine ants. In 1995, Bilchev and Parmee [4] proposed the continuous ant colony algorithm for the first time; however, what they investigated actually was the genetic algorithm based on the ant colony algorithm. $\mathrm{Li}$ and $\mathrm{Wu}[5]$ proposed an adaptive ant colony system algorithm for continuous-space optimization problems. Chen et al. [6] presented a continual domain ant colony algorithm based on overlapping mutation operations. Dréo and Siarry $[7,8]$ proposed a continuously interacting ant colony algorithm based on an intensive nonhierarchical process. Chen [9] proposed a method that approximates the variance of continuous functions with discrete points. Zhang and Huang [10] proposed a continual domain ant colony algorithm for solving multiobjective function optimizations with constraints. In general ant colony algorithms, however, the information difference is achieved through genetic manipulation, which easily causes premature events. To address the premature defect, various niching approaches, usually called 
niching techniques, were recently developed. Among these niching methods (including crowding [11], fitness-sharing [12], clearing [13], and clustering-based niching methods [14]), fitness sharing is a well-known niching technique that offers a variety of modified schemes [15-17]. Niching, as an evolutionary computation concept, was first formally applied to the genetic algorithms (GAs). However, niching has also been applied to other algorithms, such as the ant colony algorithm, and promising results were obtained [18, 19]. Although niche ant colony algorithms (NACA) were adopted to deal with a number of optimization problems, no relevant report is yet available in the field of parameter inversion. In this paper, NACA based on the fitness-sharing principle was applied in the inversion of the parameters of the Biot model and provided improved results.

The layout of this work is as follows. The mathematical model of Biot theory is presented in Section 2 and the improved niche ant colony algorithm is introduced in detail in Section 3. Section 4 shows a computer animation of parameters inversion of the Biot model. Finally, the conclusion is presented in the last section.

\section{Mathematics Model}

One-dimensional two-phase medium kinetic equations can be expressed as

$$
\begin{gathered}
\frac{\partial}{\partial x}\left[\left(\lambda+2 \mu+\alpha^{2} Q\right) \frac{\partial u}{\partial x}\right]+\frac{\partial}{\partial x}\left[(\alpha Q) \frac{\partial w}{\partial x}\right]=\rho \ddot{u}+\rho_{f} \ddot{w} \\
\frac{\partial}{\partial x}\left[(\alpha Q) \frac{\partial u}{\partial x}\right]+\frac{\partial}{\partial x}\left[(Q) \frac{\partial w}{\partial x}\right]=\rho_{f} \ddot{u}+m \ddot{w}+r \dot{w},
\end{gathered}
$$

where $\lambda, \mu$ are the Lame coefficients, $Q$ is the compressibility of pore fluid, and $\alpha$ is the effective stress parameter; $m, r$ are the coefficients introduced by Biot model, $m=\rho_{f} / \varphi$ and $r=$ $\eta / \kappa$, where $\kappa$ is permeability and $\eta$ is viscosity coefficient; $u$ is the displacement of solid skeleton, $w$ is fluid displacement relative to solid skeleton, which can be written as $w=\varphi(U-$ $u$ ), in which $U$ represents fluid displacement in pores, and $\rho$ is the bulk density of the solid-fluid mixture.

The relations of these parameters can be expressed as follows:

$$
\begin{gathered}
\lambda=K-\frac{2}{3} \mu, \quad \rho=(1-\varphi) \rho_{s}+\varphi \rho_{f}, \\
\alpha=1-\frac{K}{K_{s}} \quad Q=\frac{K_{s}^{2}}{k\left(1+\varphi\left(K_{s} / K_{f}-1\right)\right)-K},
\end{gathered}
$$

where $K, K_{f}, K_{s}$ represent the bulk change modulus of solid skeleton, fluid, solid, respectively; $\rho_{f}, \rho_{s}$ are the fluid and solid densities, respectively. Equations (1) describe the elastic wave propagation in the two-phase medium and form the forward problem with some appropriate boundary conditions and initial conditions. Knowing the corresponding parameters, the wave field and the seismograms can be obtained from the forward problem by some methods. The seismograms can be regarded as the additional conditions, and the inversion problem is to estimate some parameters from (1) with specific boundary conditions, initial conditions, and additional conditions.

In 1984, Simon et al. [20] presented a one-dimensional analytical solution for the situation, where the solid and fluid materials satisfy Biot's dynamic compatibility condition under step load and impulsive loading. It is assumed that flow occurs freely (full drainage) through the surface $(x=0)$ of the medium. The boundary conditions can be expressed as

$$
\sigma^{\prime}(0, t)=F(t), \quad \pi(0, t)=0,
$$

where $\sigma^{\prime}$ denotes the stress, $F(t)$ is an arbitrary function of time describing the prescribed surface traction, and $\pi$ is the pore fluid pressure.

The initial conditions are

$$
u(x, 0)=\dot{u}(x, 0)=w(x, 0)=\dot{w}(x, 0)=0 .
$$

Simon obtained the analytic solution of previous initialboundary value problem using Laplace transformation and numerical inversion for some material properties. The solutions are

$$
\begin{array}{r}
w(\xi, \tau)=\frac{\alpha}{(1-\alpha \chi) \sqrt{a} \int_{0}^{\tau} f(\tau-\bar{\tau}) e^{-b \bar{\tau} / 2 a}} \\
\cdot I_{0}\left[\frac{b \sqrt{\bar{\tau}^{2}-a \xi}}{2 a}\right] H(\bar{\tau}-\xi \sqrt{a}) d \bar{\tau} \\
u(\xi, \tau)=-\int_{0}^{\tau} f(\tau-\bar{\tau}) \cdot H(\bar{\tau}-\xi) d \bar{\tau}-\chi w(\xi, \tau),
\end{array}
$$

where $\xi=x / \rho k V_{c}, \tau=t / \rho k, f(\tau)=\left(k / V_{c}\right) f(t), \chi=\rho_{f} / \rho$, $\gamma=\chi / \varphi$,

$$
\begin{gathered}
V_{c}=\sqrt{\frac{\lambda+2 \mu+\alpha^{2} Q}{\rho}}, \quad k=\frac{Q}{\lambda+2 \mu+\alpha^{2} Q}, \\
a=\frac{\left(\gamma-\chi^{2}\right)}{\left(k-\chi^{2}\right)}, \\
b=\frac{1}{\left(k-\chi^{2}\right)},
\end{gathered}
$$

where $I_{0}[z]$ is the modified Bessel function of zero order and $H(\tau)$ is unit step function.

\section{Niche Ant Colony Algorithm (NACA)}

The concept of the niche was first introduced in [21], although the definition was slightly altered since its introduction. In [22], each species was believed to occupy its own unique niche within the environment. The analogy in nature is that, within the environment, different subspaces (niches) exist, which can support different types of life (species or organisms). The number of organisms contained within a niche is determined both by the fertility of the niche (or carrying capacity) and by the efficiency of each organism in exploiting niche fertility. If an excessive number of organisms exist for a given niche, food will be inadequate, and the least 
efficient organisms will die. Conversely, if few organisms exist in a very fertile niche, these organisms will quickly reproduce to exploit fully the capability of the niche to support life.

In a computational sense, niching may permit a more effective use of available resources through a search algorithm by either implicitly or explicitly dividing and searching different areas of the search space in parallel. Such an automatic resource reallocation is useful in preserving population diversity for an extended search time. Niching techniques have been proven particularly useful in problems that require multiple solutions to be found for a search algorithm, such as multimodal and multiobjective optimization problems.

\subsection{Niche Ant Colony Algorithm Based on the Fitness Sharing.} Niche formation, specifically relative to fitness sharing, was explained by Goldberg and Richardson [12] by using a variation of the $k$-armed bandit problem. The niching algorithm was applied to find and preserve multiple solutions in GAs. With the fitness-sharing approach, the search landscape is modified by reducing the payoff in densely populated regions. This approach reduces the fitness of an individual by an amount that is proportional to the number of similar individuals in the population. On the basis of the same theory, we applied the fitness-sharing algorithm to NACA and proposed an improved NACA based on fitness sharing called INACA.

Similarly, in this paper, a sharing function is defined to calculate the niche counts, which are further used immediately prior to the selection operation to derate the fitness of individuals in densely populated subspaces as follows:

$$
\operatorname{Sh}\left(d_{i j}\right)= \begin{cases}1-\left(\frac{d_{i j}}{\sigma}\right)^{\alpha} & d_{i j}<\sigma \\ 0 & \text { otherwise, }\end{cases}
$$

where $\sigma$ is the niche radius that is given by the formula

$$
\sigma=\left(\frac{1}{(2 \sqrt[n]{q})}\right) \sqrt{\sum_{\kappa=1}^{n}\left(x_{\kappa}^{u}-x_{\kappa}^{l}\right)^{2}}
$$

where $q$ is the number of niches, $n$ is the dimension of the feasible region, $\left\{x_{\kappa}^{u}, x_{\kappa}^{l}\right\}$ denotes the upper and lower bounds of the $k$ th dimension of the feasible region, $d_{i j}$ is the distance between individuals $i$ and $j$, and $i, j=1,2, \ldots N$. The sharing function describes the similarity among different individuals: $\operatorname{Sh}\left(d_{i j}\right)=1$ indicates that $i$ and $j$ are the same individuals or $d_{i j}=0$, whereas $\operatorname{Sh}\left(d_{i j}\right)=0$ suggests that $i$ and $j$ belong to different niches. In formula (7), $\alpha$ controls the shape of the sharing function, and the triangular sharing function is obtained with $\alpha=1$. The similarity among individuals for real coded evolutionary algorithms is computed through the Euclidean distance in real-valued space.

For each individual $i$, some individuals are measured to be similar by $\sigma$. The raw fitness $f_{i}$ is to be shared with such individuals. The niche count $m_{i}$ is given by

$$
m_{i}=\sum_{j=1}^{N} \operatorname{Sh}\left(d_{i j}\right)
$$

where $N$ denotes the population size. The value of $m_{i}$ is equivalent to the number of individuals around the $i$ th individual. A large $m_{i}$ value indicates that more individuals surround the $i$ th individual. The shared fitness of individual $i$, with raw fitness $f_{i}$, is given by

$$
f_{i}^{\prime}=\frac{f_{i}}{m_{i}} .
$$

Through fitness sharing, the replicas and the offspring of an individual are produced inversely proportional to the similar ones in the same niche. Even elitists could not take over the population, which means that the fitness-sharing scheme is capable of counterbalancing the genetic drift. Thus, the fitness-sharing technique allows the exploration and exploitation of fitness landscape by favoring the formation of stable subpopulations. With properly parameterized fitness sharing (population size $N$ and niche radius $\sigma$ ), the niching equilibrium is eventually reached, where all individuals are distributed among niches according to their fitness, and all species of the identified niches are maintained to the final population.

A general pseudocode representation is provided as Algorithm 1.

\section{Inversion Example}

In this section, we perform the inversion of porosity and permeability based on the previous Biot model with the improved niche ant colony algorithm. The true values of twophase medium materials [23] are $\alpha=0.667, \eta=0.001$, $k=0.004883 \mathrm{~m}^{2}, \rho=306 \mathrm{~kg} / \mathrm{m}^{3}, \chi=0.9730$, and $\varphi=0.333$. We choose the displacement responses $u^{*}(0, t)$ and $w^{*}(0, t)$ at the surface $(x=0)$ as the measurements at receiver locations on the boundary, that is, the additional conditions, which can be obtained from formula (5) based on the previous true values. The normal stress is $\sigma^{\prime}(0, t)=F(t)$ at the surface, where $F(t)$ is taken to be a step function as follows

$$
F(t)=\sigma_{0}^{\prime} H(t)
$$

where $H(t)$ is Heaviside function and $\sigma_{0}^{\prime}=1000 \mathrm{kPa}$. We choose to inverse the parameters at the time $t=0.03 \mathrm{~s}$. In fact, the inverse problem can be formulated as an optimization problem. The objective function is defined as:

$$
E=\operatorname{abs}\left(u(0, t)-u^{*}(0, t)\right)+\operatorname{abs}\left(w(0, t)-w^{*}(0, t)\right) .
$$

Setting the number of niches to 2 , the number of individuals is 10 , the transition probability is 0.97 , the pheromone evaporation factor is 0.2 , and the radius is still calculated according to formula (9). We initially performed the inversion of a single parameter. Table 1 shows the results. The table shows that the inversion precision of these parameters is very high and that the relative error of the single parameter inversion can be maintained at less than $0.4 \%$; particularly the relative error of porosity can achieve less than $0.02 \%$. These results illustrate that INACA is valid for single parameter inversion in the Biot model. 
procedure Initialization

(i) Parameters Initialization:: such as population size $\mathbf{p}_{\text {size }}$, the global transition probability $\mathbf{P}_{0}$, the evaporation coefficient of pheromone $\mathbf{p}_{\mathrm{ec}}$, the proportional factor $\mathbf{k}$, the feasible region [start, end], and the radius of niche $\sigma$ (Obtained from the Formula (8));

(ii) Population Initialization

for $i=1$ to $\mathbf{p}_{\text {size }}$ do

$\mathbf{X}[\mathbf{i}]=\mathbf{s t a r t}+($ end - start $) \times \operatorname{rand}(\mathbf{1}) ;($ The initial position of ants $)$

the optimization function)

FitnessValue $[\mathbf{i}]=\mathbf{k} \cdot \mathbf{f}(\mathbf{X}[\mathbf{i}])$; (The fitness value before sharing, where $f$ is

end procedure

while stopping criterion not met do

De-rate quality;

Calculate transition probability;

Update positions of ants (in the feasible region);

Update pheromones;

end while

procedure De-rate quality

for $i=1$ to $\mathbf{p}_{\text {size }}$ do

NicheCount $=0$

for $j=1$ to $\mathbf{p}_{\text {size }}$ do

$d=\operatorname{distance}\left(\mathbf{p}_{\mathbf{i}}, \mathbf{p}_{\mathbf{j}}\right)$

if $\mathbf{d}<\boldsymbol{\sigma}$ then

Share Value $=\left(1-(\mathbf{d} / \sigma)^{\alpha}\right)$

else

Share Value $=0$

endif

NicheCount $=$ NicheCount + ShareValue

end for

FitnessValue-Modified $=$ FitnessValue/NicheCount $($ The fitness value after

sharing)

end for

end procedure

procedure Calculate transition probability

$T[i]=k$ /FitnessValue-Modified; (The initial pheromone)

$T \_$Best $=\operatorname{Max}(T)$;

For $i=1$ to $\mathbf{p}_{\text {size }}$ do

$\operatorname{Prob}[i]=\left(T \_\right.$Best $\left.-T\right) / T \_$Best

end do

For $i=1$ to $\mathbf{p}_{\text {size }}$ do

If $\operatorname{Prob}[i]<P_{0}$

else

Temp $=X[i]+$ min_step $*(\operatorname{rand}(1)-0.5)$

Temp $=X[i]+$ max_step $*(\operatorname{rand}(1)-0.5)$

endif

if Temp $<$ start

Temp = start;

elseif Temp > end

endif

Temp = end 1 ;

end do

end procedure

procedure Update positions of ants and pheromones

For $i=1$ to $\mathbf{p}_{\text {size }}$ do

if $f($ Temp $) /$ NicheCount $<f(X[i]) /$ NicheCount

$X[i]=$ Temp

FitnessValue-Modified $=f(X[i]) /$ NicheCount;

endif

$T[i] \_$new $=\left(1-p_{\mathrm{ec}}\right) * T[i]+k / f(X[i]) ;$ (Update pheromones)

end do

end procedure

Algorithm 1: Niching ant colony optimization based on the fitness-sharing principle. 
TABLE 1: The single parameter inversion results based on Biot model.

\begin{tabular}{lcccc}
\hline Parameter & True value & Inversion value & Absolute error & Relative error $(\%)$ \\
\hline Porosity & 0.333000 & 0.333066 & 0.000066 & 0.019820 \\
Permeability & 0.004883 & 0.004868 & 0.000015 & 0.307188 \\
\hline
\end{tabular}

TABLE 2: The double parameters inversion results based on the Biot model.

\begin{tabular}{lcccc}
\hline Parameter & True value & Inversion value & Absolute error & Relative error $(\%)$ \\
\hline Porosity & 0.333000 & 0.332376 & 0.000624 & 0.187387 \\
Permeability & 0.004883 & 0.004526 & 0.000357 & 7.311079 \\
\hline
\end{tabular}

TABLE 3: The inversion results of single parameter with 5\% noise.

\begin{tabular}{lcccc}
\hline Parameter & True value & Inversion value & Absolute error & Relative error $(\%)$ \\
\hline Porosity $\varphi$ & 0.333000 & 0.333084 & 0.000084 & 0.025225 \\
Permeability $k$ & 0.004883 & 0.004902 & 0.000019 & 0.389105 \\
\hline
\end{tabular}

TABLE 4: The inversion results of two parameters with 5\% noise.

\begin{tabular}{lcccc}
\hline Parameter & True value & Inversion value & Absolute error & Relative error $(\%)$ \\
\hline Porosity $\varphi$ & 0.333000 & 0.332193 & 0.000807 & 0.242342 \\
Permeability $k$ & 0.004883 & 0.004465 & 0.000418 & 8.560311 \\
\hline
\end{tabular}

The foregoing results demonstrate that the INACA has a high precision in single parameter inversion, but in actual exploration of stratum we maybe need to predict two or more parameters. We then conducted an inversion of double parameters based on the Biot model; the inversion results are listed in Table 2. For double parameters inversion, the inversion result of the permeability is slightly weak, but the relative error can still be maintained at less than $7.5 \%$.

In order to verify the noise resistance of the improved ant colony algorithm, we added 5\% noise on the seismograms by the following ways:

$$
\begin{aligned}
& u_{\delta}^{*}(0, t)=u^{*}(0, t)(1+\delta \times \operatorname{random}(x, t)), \\
& w_{\delta}^{*}(0, t)=w^{*}(0, t)(1+\delta \times \operatorname{random}(x, t)),
\end{aligned}
$$

where $\delta=0.05$. In this process, the parameter values are the same as the foregoing examples. Tables 3 and 4 show the inversion results with $5 \%$ noise for a single parameter and two parameters, respectively. The tables show that the inversion results are satisfactory.

\section{Conclusion}

We numerically studied the use of INACA for parameter estimation based on the Biot model in a fluid-saturated porous medium. As seen in the examples, the method proposed in this paper was successful at inverting porosity and permeability. The relative errors of single parameter inversion can be maintained at less than $0.4 \%$, and the relative errors of two-parameter (porosity and permeability) inversion were slightly weak; the relative error can still be maintained at less than $7.5 \%$. These results demonstrate that the improved niche ant colony algorithm is well suited for solving this ill-posed problem. Also shown in this paper is the result that the improved niche ant colony algorithm has a better antinoise property for the two-parameter estimation. With $5 \%$ noise, the results obtained with the improved niche ant colony algorithm are satisfactory.

\section{Conflict of Interests}

The authors declare that there is no conflict of interests regarding the publication of this paper.

\section{Acknowledgments}

This work was supported by the National Science Foundation of China, under Grant no. 41004052, and the Specialized Research Fund for the Doctoral Program of Higher Education of China, under Grant no. 20102302120072.

\section{References}

[1] M. A. Biot, "Theory of propagation of elastic waves in a fluidsaturated porous solid, Low-frequency range," Journal of the Acoustical Society of America, vol. 28, pp. 168-178, 1956.

[2] M. A. Biot, "Theory of propagation of elastic waves in a fluidsaturated porous solid, Higher-frequency range," Journal of the Acoustical Society of America, vol. 28, pp. 179-191, 1956.

[3] A. Colorni, M. Dorigo, and V. Maniezzo, "Distributed optimization by ant colonies," in Proceedings of the 1st EuroPean Conference on Artificial Life, pp. 134-142, 1991.

[4] G. A. Bilchev and I. C. Parmee, "The ant colony metaphor for searching continuous spaces," Lecture Notes in Computer Science, vol. 993, pp. 25-39, 1995. 
[5] Y. J. Li and T. J. Wu, "Adaptive ant colony system algorithm for continuous-space optimization problems," Pattern Recognition and Artificial Intelligence, vol. 14, no. 4, pp. 423-427, 2001.

[6] L. Chen, J. Shen, and L. Qin, "Method for solving optimization problem in continuous space using ant colony algorithm," System Engineering Theory and Practice, vol. 23, no. 3, pp. 4853, 2003.

[7] J. Dréo and P. Siarry, "Continuous interacting ant colony algorithm based on dense heterarchy," Future Generation Computer Systems, vol. 20, no. 5, pp. 841-856, 2004.

[8] J. Dréo and P. Siarry, "An ant colony algorithm aimed at dynamic continuous optimization," Applied Mathematics and Computation, vol. 181, no. 1, pp. 457-467, 2006.

[9] Y. Chen, "Ant colony system for continuous function optimization," Journal of Sichuan University, vol. 36, pp. 117-120, 2004.

[10] Y.-D. Zhang and S.-B. Huang, "Ant colony algorithm for solving multiobjective optimization problems," Control and Decision, vol. 20, no. 2, pp. 170-178, 2005.

[11] S. W. Mahfoud, "Crowding and preselection revisited," in Parallel Problem Solving From Nature 2 (PPSN2), R. Männer and B. Manderick, Eds., pp. 27-36, North-Holland, Amsterdam, The Netherlands, 1992.

[12] D. E. Goldberg and J. Richardson, "Genetic algorithms with sharing for multi-model function optimization," in Proceedings of the 2nd Conference on Genetic Algorithms, pp. 41-47, Lawrence Erlbaum Associates, Hillsdale, NJ, USA, 1987.

[13] A. Petrowski, "Clearing procedure as a niching method for genetic algorithms," in Proceedings of the IEEE International Conference on Evolutionary Computation (ICEC '96), pp. 798803, IEEE, Los Alamitos, Calif, USA, May 1996.

[14] F. Streichert, G. Stein, H. Ulmer, and A. Zell, "A clustering based niching EA for multimodal search spaces," in Proceedings of the International Conference Evolution Artificielle, vol. 2936 of Lecture Notes in Computer Science, pp. 293-304, Springer, 2003.

[15] B. L. Miller and M. J. Shaw, "Genetic algorithms with dynamic niche sharing for multimodal function optimization," in Proceedings of the IEEE International Conference on Evolutionary Computation (ICEC '96), IlliGAL Report No. 95010, pp. 786791, IEEE Press, Piscataway, NJ, USA, May 1996.

[16] B. Sareni and L. Krähenbühl, "Fitness sharing and niching methods revisited," IEEE Transactions on Evolutionary Computation, vol. 2, no. 3, pp. 97-106, 1998.

[17] A. Della Cioppa, C. De Stefano, and A. Marcelli, "On the role of population size and niche radius in fitness sharing," IEEE Transactions on Evolutionary Computation, vol. 8, no. 6, pp. 580-592, 2004.

[18] D. Angus, "Niching for population-based ant colony optimization," in Proceedings of the 2nd International IEEE Conference on e-Science and Grid Computing, Workshop on Biologicallyinspired Optimisation Methods for Parallel and Distributed Architectures: Algorithms, Systems and Applications, 2006.

[19] D. Angus, "Crowding population-based ant colony optimisation for the multi-objective travelling salesman problem," in Proceedings of the 1st IEEE Symposium of Computational Intelligence in Multicriteria Decision Making (MCDM '07), pp. 333-340, IEEE, Piscataway, NJ, USA, April 2007.

[20] B. R. Simon, O. C. Zienkiewicz, and D. K. Paul, "An analytical solution for the transient response of saturated porous elastic solids," International Journal for Numerical and Analytical Methods in Geomechanics, vol. 8, no. 4, pp. 381-398, 1984.

[21] J. Grinnell, "The niche relationship of the california thrasher," Auk, vol. 34, pp. 427-433, 1917.
[22] G. E. Hutchinson, "Concluding remarks, cold spring harbor symposium," Quantitative Biology, vol. 22, pp. 415-427, 1957.

[23] D. H. Yang and X. H. Chen, "BISQ model for fluid-filled, porous medium," Oil Geophysical Prospecting, vol. 36, no. 2, pp. 146-159, 2001. 


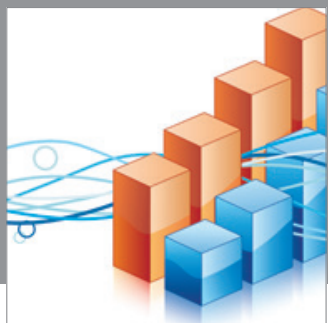

Advances in

Operations Research

mansans

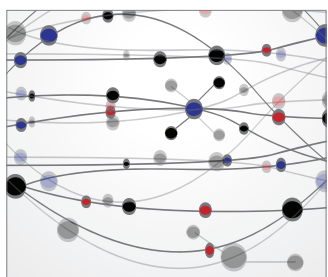

The Scientific World Journal
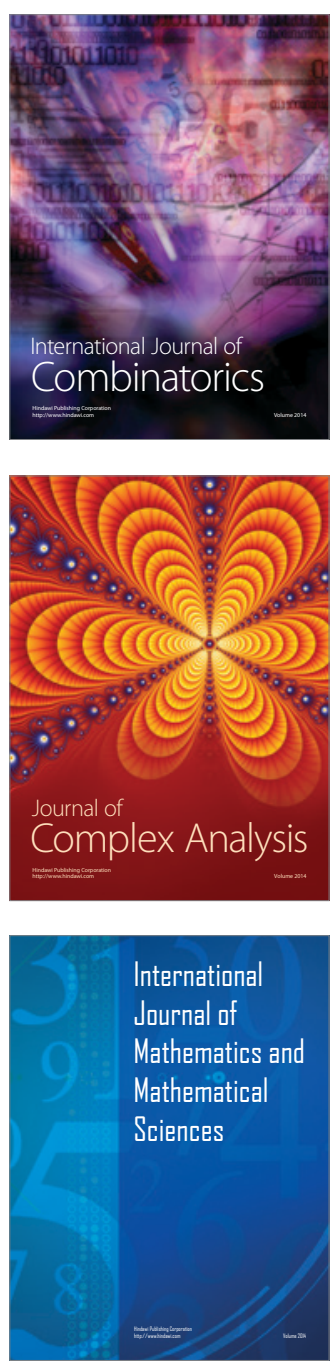
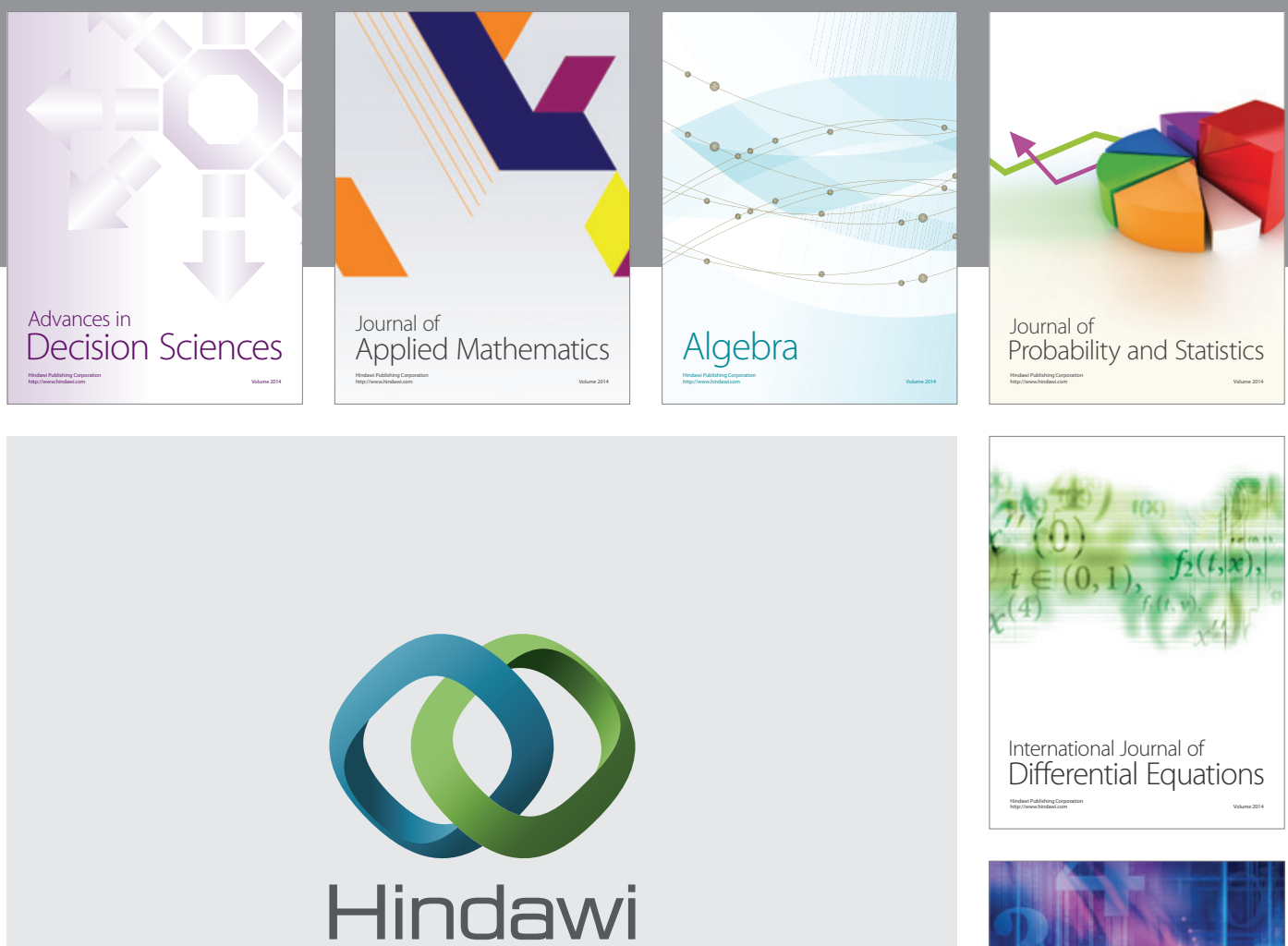

Submit your manuscripts at http://www.hindawi.com
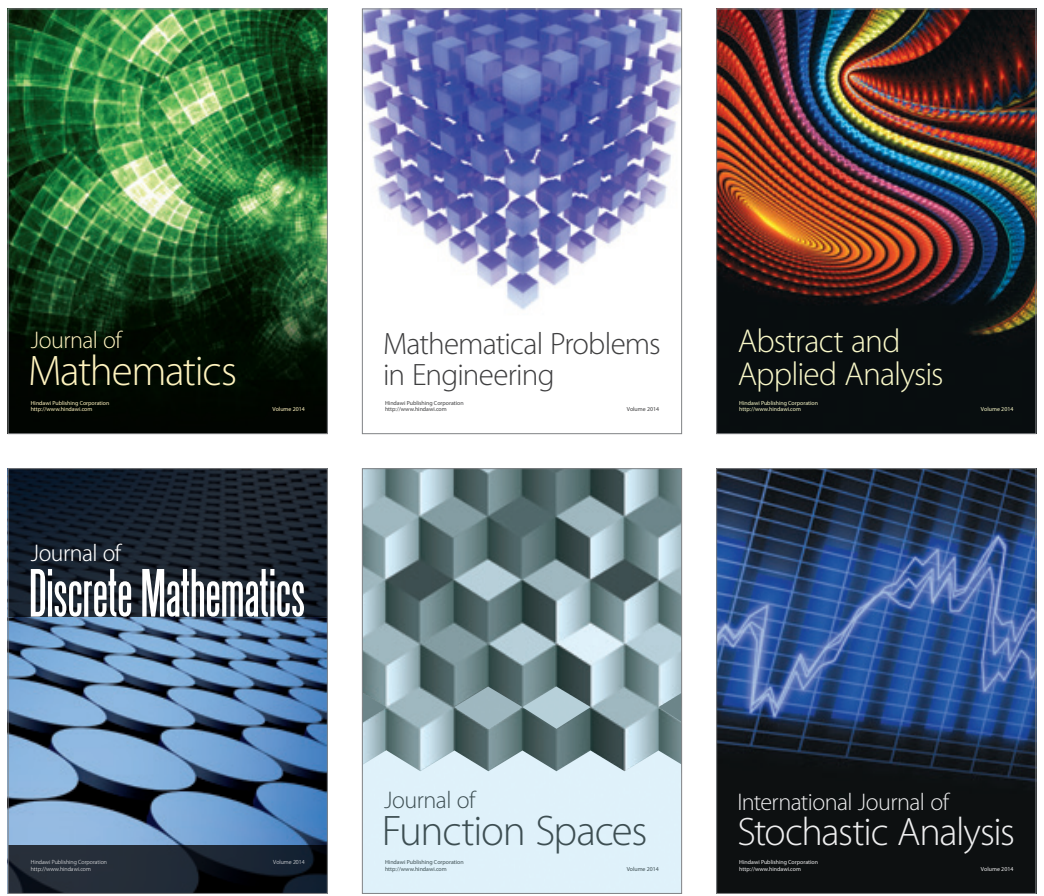

Journal of

Function Spaces

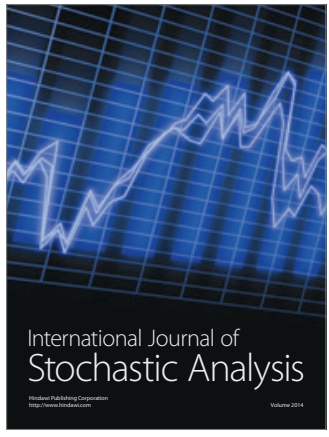

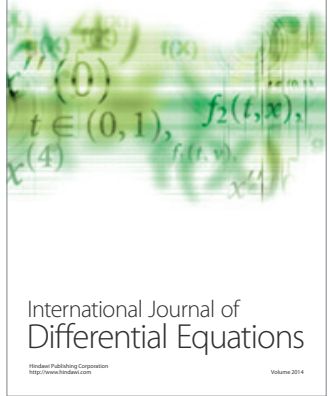
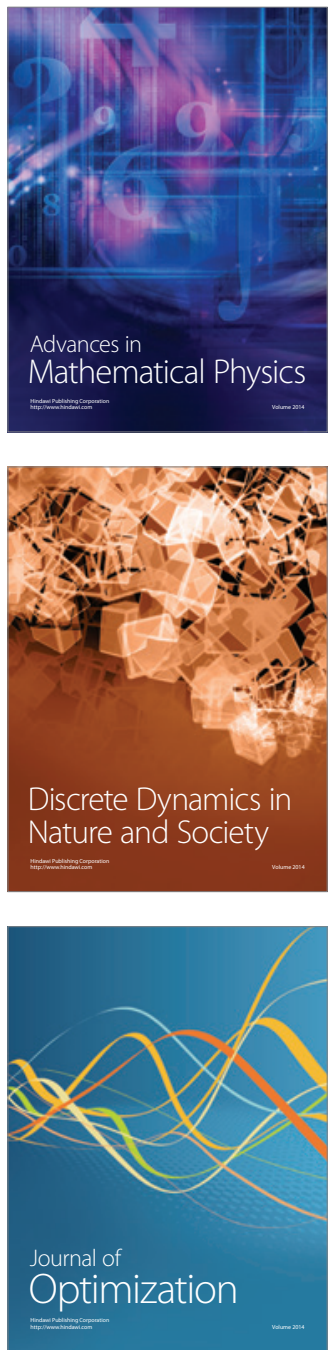